\title{
Surface correction to Landau diamagnetism
}

\author{
Roland E. Allen \\ Department of Physics, Texas A\&M University, College Station, Texas 77843 \\ (Received 4 December 1974)
}

\begin{abstract}
For a semi-infinite free-electron model with a magnetic field applied perpendicular to the surface, we show that $\chi_{d}^{s}=-\chi_{p}^{s} / 3$, where $\chi_{d}^{s}$ and $\chi_{p}^{s}$ are, respectively, the surface corrections to the Landau diamagnetism and the Pauli paramagnetism. The total surface contribution to the susceptibility is $\chi^{s}$ $=(2 / 3)\left(\mu_{B}^{2} / \pi^{2}\right) A\left[\gamma\left(k_{F}\right)-\pi / 4\right]$, where $\gamma\left(k_{F}\right)$ is the phase shift for $k_{z}=k_{F}$. Since $\langle\gamma\rangle=\pi / 4$ and $\left.\gamma\left(k_{F}\right)\right\rangle\langle\gamma\rangle$, the surface contributions to the magnetic susceptibility and electronic heat capacity are positive.
\end{abstract}

In a classic paper, ${ }^{1}$ Landau calculated the diamagnetic orbital susceptibility of the free-electron gas, $\chi_{d}^{b}$, and found that

$$
\chi_{d}^{b}=-\frac{1}{3} \chi_{p}^{b},
$$

where $\chi_{p}^{b}$ is the (Pauli) paramagnetic spin susceptibility. In the present paper, we consider the effect of a surface perpendicular to the magnetic field, and we show that

$$
\chi_{d}^{s}=-\frac{1}{3} \chi_{p}^{s},
$$

where $\chi_{d}^{s}$ and $\chi_{p}^{s}$ are, respectively, the surface corrections to the diamagnetic and paramagnetic susceptibilities.

We will consider a slab of thickness $L$. The magnetic field $\overrightarrow{\mathrm{B}}$ is applied along the $z$ axis, which is normal to the surface. The electronic states are the Landau orbitals, ${ }^{2}$ which are characterized by a wave vector $k_{z}$ and a quantum number $n$. There are $A e B / 2 \pi c \hbar$ states with the same $k_{z}$ and $n$, and the same energy

$$
\epsilon_{n}\left(k_{z}\right)=\left(\hbar^{2} / 2 m\right) k_{z}^{2}+2 \mu_{B} B\left(n+\frac{1}{2}\right),
$$

where $A$ is the total area of both surfaces and spin degeneracy has been included. The "grand canonical potential" is given by

$$
\begin{aligned}
\Omega= & -k_{B} T\left(\frac{A e B}{2 \pi c \hbar}\right) \sum_{k_{z}} \sum_{n=0}^{\infty} \\
& \times \ln \left(1+\exp \left\{\left[\epsilon_{F}-\epsilon_{n}\left(k_{z}\right)\right] / k_{B} T\right\}\right) .
\end{aligned}
$$

The following version of the Euler-Mac Laurin formula is useful ${ }^{2}$ :

$$
\sum_{n=0}^{\infty} F(n)=\int_{-1 / 2}^{\infty} F(n) d n+\frac{1}{24} F^{\prime}\left(-\frac{1}{2}\right)+\ldots,
$$

where $F(n)$ is the function summed in (4). Since

$$
F^{\prime}(n)=-\left(2 \mu_{B} B / k_{B} T\right) f\left(\epsilon_{n}\left(k_{z}\right)\right),
$$

where $f$ is the Fermi-Dirac distribution function, we have

$$
\Omega=-k_{B} T \frac{A e}{4 \pi c \hbar \mu_{B}} \sum_{k_{z}} \int_{0}^{\infty} \ln [1
$$

$$
\begin{aligned}
& \left.+\exp \left(\left\{\epsilon_{F}-\left[\epsilon\left(k_{z}\right)+\epsilon\right]\right\} / k_{B} T\right)\right] d \epsilon \\
& +\frac{1}{24} \frac{A e \mu_{B}}{\pi c \hbar} B^{2} \sum_{k_{z}} f\left(\epsilon\left(k_{z}\right)\right),
\end{aligned}
$$

where $\epsilon\left(k_{z}\right) \equiv \hbar^{2} k_{z}^{2} / 2 m$, and we have neglected terms of order $B^{3}$ and higher. The magnetic moment is

$$
\begin{aligned}
& M=-\frac{\partial \Omega}{\partial B} \\
& =-\frac{1}{6} \frac{m A \mu_{B}^{2}}{\pi \hbar^{2}} B \sum_{k_{z}} f\left(\epsilon\left(k_{z}\right)\right),
\end{aligned}
$$

since $\mu_{B}=e \hbar / 2 m c$. In a previous paper, ${ }^{3}$ we obtained a result which reduces to

$$
\sum_{k_{z}} f\left(k_{z}\right)=\frac{L}{\pi} \int_{0}^{\infty} f\left(k_{z}\right) d k_{z}+\frac{2}{\pi} \int_{0}^{\infty} f\left(k_{z}\right) d \gamma\left(k_{z}\right)-\frac{1}{2} f(0),
$$

in the case of a free-electron model with no surface states. Terms of order $L^{-2}$ and smaller have been neglected. We integrate by parts, and use the fact that $f(\infty)=0$ and $\gamma(0)=0$, to obtain

$$
\begin{aligned}
\sum_{k_{z}} f\left(\epsilon\left(k_{z}\right)\right)= & -\frac{L}{\pi} \int_{0}^{\infty} k_{z} f^{\prime}\left(\epsilon\left(k_{z}\right)\right) d \epsilon\left(k_{z}\right)-\frac{2}{\pi} \\
& \times \int_{0}^{\infty} \gamma\left(k_{z}\right) f^{\prime}\left(\epsilon\left(k_{z}\right)\right) d \epsilon\left(k_{z}\right)-\frac{1}{2} f(0) .
\end{aligned}
$$

We now assume $k_{B} T \ll \epsilon_{F}$, so that $f(0) \approx 1$ and $-f^{\prime}(\epsilon)$ $\approx \delta\left(\epsilon-\epsilon_{F}\right)$.

$$
\sum_{k_{z}} f\left(\epsilon\left(k_{z}\right)\right)=\frac{L}{\pi} k_{F}+\frac{2}{\pi} \gamma\left(k_{F}\right)-\frac{1}{2} .
$$

Then

$$
M=-\frac{1}{3}\left(m \mu_{B}^{2} / \pi^{2} \hbar^{2}\right)\left\{V k_{F}+A\left[\gamma\left(k_{F}\right)-\frac{1}{4} \pi\right]\right\} B .
$$

In atomic units, with $m / \hbar^{2}=1$,

$$
\begin{aligned}
\chi_{d} & \equiv \frac{\partial M}{\partial B} \\
& =-\frac{1}{3}\left(\mu_{B}^{2} / \pi^{2}\right)\left\{V k_{F}+A\left[\gamma\left(k_{F}\right)-\frac{1}{4} \pi\right]\right\} .
\end{aligned}
$$

Here, as in our previous paper, ${ }^{4}$ we have defined $\chi$ to be the derivative of the magnetic moment, 
rather than the magnetization. The surface contribution is

$$
\chi_{d}^{s}=-\frac{1}{3}\left(\mu_{B}^{2} / \pi^{2}\right) A\left[\gamma\left(k_{F}\right)-\frac{1}{4} \pi\right] .
$$

The surface contribution to the spin susceptibility is $^{4}$

$$
\chi_{p}^{s}=\frac{\mu_{B}^{2}}{\pi^{2}} A\left[\gamma\left(k_{F}\right)-\frac{1}{4} \pi\right],
$$

so we have the result (2) given above.

The total susceptibility is

$$
\chi^{s}=\frac{2}{3}\left(\mu_{B}^{2} / \pi^{2}\right) A\left[\gamma\left(k_{F}\right)-\frac{1}{4} \pi\right] .
$$

Also, the surface electronic heat capacity is ${ }^{4}$

$$
C_{\mathrm{e} 1}^{s}=\frac{1}{3} A\left[\gamma\left(k_{F}\right)-\frac{1}{4} \pi\right] k_{B}^{2} T .
$$

The sum rule of Sugiyama ${ }^{5}$ and Langreth ${ }^{6}$ states that

$$
\langle\gamma\rangle=\frac{1}{4} \pi,
$$

where $\langle\gamma\rangle$ is an average over $k_{z} \leq k_{F}$. We expect $\gamma\left(k_{z}\right)$ to increase with $k_{z}$, because electrons with larger kinetic energy normal to the surface should penetrate further into the vacuum. ${ }^{7}$ Then we have

$$
\gamma\left(k_{F}\right)>\frac{1}{4} \pi, \quad C_{\mathrm{e} 1}^{s}>0, \quad \chi^{s}>0 .
$$

Measurements of susceptibilities and heat capacities of very small metallic particles, or very thin films, would be of interest in testing the qualitative conclusions of (21). Of course, in real materials there will be large corrections produced by electron correlations and crystallinity, effects that are omitted in the present model of noninteracting free electrons.

As can be seen from (15), or Eqs. (9) and (10) of Ref. 4, the ratio of surface to volume contributions is

$$
\left(A / V k_{F}\right)\left[\gamma\left(k_{F}\right)-\frac{1}{4} \pi\right] \sim 1 / k_{F} L
$$

so that the fractional change in $C_{\mathrm{el}}$ and $\chi$ due to a surface should be of the same order of magnitude as the ratio of the number of surface atoms to the number of volume atoms.
${ }^{1}$ L. D. Landau, Z. Phys. 64, 629 (1930).

${ }^{2} \mathrm{~A}$. A. Abrikosov, Introduction to the Theory of Normal Metals (Academic, New York, 1972), pp. 177-182.

${ }^{3}$ R. E. Allen, Solid State Commun. (to be published).

${ }^{4}$ V. E. Kenner and R. E. Allen, Phys. Rev. B $\underline{11}, 2858$ (1975).
${ }^{5}$ A. Sugiyama, J. Phys. Soc. Jpn. 15, 965 (1960).

${ }^{6}$ D. C. Langreth, Phys. Rev. B $\underline{5}, 2842$ (1972).

${ }^{7}$ Sugiyama, in Ref. 5, has, in fact, plotted $\gamma\left(k_{z}\right)$ vs $k_{z}$ for various model potentials (see his Figs. 3 and 5), and in every case $\gamma\left(k_{z}\right)$ increases monotonically with $k_{z}$. 